**THIS PRE-PRINT IS A WORKING PAPER THAT HAS NOT BEEN PEER-REVIEWED**

\title{
A double-shot against COVID-19? Hope for a vaccine increases mitigation intentions and emotional well-being by reducing fatalism
}

\author{
Joseph Hayes and Laura Clerk \\ Department of Psychology, Acadia University
}

[word count (excluding abstract, statement of relevance, methods, results, and references): 1997]

\section{Author Note}

The materials used in the research, as well as de-identified data files and analysis scripts, are posted at https://osf.io/uj6d2/. We have no known conflict of interest to disclose. Financial support for this project was provided by the Acadia University Research Fund and a Harrison McCain Emerging Scholar Grant awarded to the first author.

Correspondence concerning this article should be addressed to Joseph Hayes, Acadia University, Department of Psychology, 18 University Ave, Wolfville, NS, B4P 2R6, Canada. Email: joseph.hayes@acadiau.ca 


\begin{abstract}
This research assessed the causal effect of hope for a COVID-19 vaccine on behavioral intentions to support mitigation efforts (e.g., social distancing) and emotional well-being during the coronavirus pandemic. Two studies $(N=1297)$ tested the hypothesis that experimentally manipulated hope for a vaccine would increase mitigation intentions and emotional well-being by reducing fatalism toward COVID-19. In Study 1, hope for the vaccine manipulated with an article that promoted (vs. undermined vs. control) the likelihood that a vaccine would be developed to stop the pandemic. In Study 2, hope was manipulated with real news emerging from vaccine developers indicating that two vaccines highly effective and were nearing ready. Both manipulations reduced COVID-19 fatalism and thereby increased mitigation intentions and emotional well-being. Study 2 furthermore found that positive vaccine news increased people's intentions to get vaccinated. Implications for COVID-19 mitigation efforts and mental health in the face of the coronavirus pandemic are discussed.
\end{abstract}

Keywords: COVID-19, Hope, Vaccine, Fatalism, Virus Mitigation, Mental Health 


\section{Statement of Relevance}

This research examined the effect of hope for a COVID-19 vaccine on behavioral intentions to follow public health orders (e.g., mask wearing, social distancing) and emotional well-being. Hope in a vaccine was experimentally manipulated by exposing people to opinions that promote (vs. undermine) the viability of a vaccine (Study 1) or to positive news about Phase-3 clinical vaccine trials (Study 2). Results show that heightened hope for a vaccine increased behavioral intentions to following mitigation protocols and emotional well-being by reducing a sense of fatalism toward the virus. Moreover, Study 2 found that positive vaccine news increased behavioral intentions to get vaccinated, also by reducing fatalism. These results have important implications for public health messaging about COVID-19 vaccines. 


\section{A double-shot against COVID-19? Hope for a vaccine increases mitigation intentions and emotional well-being by reducing fatalism}

Since March 2020, the world has been gripped by a deadly coronavirus pandemic. At the time of writing (November 2020), nearly 1.5 Million people have died (Worldometer, 2020). Efforts to stem the coronavirus disease (i.e., COVID-19) have included everything from social distancing, mask-wearing and reducing social contacts, to full-on lockdowns and stay-at-home orders. And yet, after more than eight months of these efforts, the spread of COVID-19 is accelerating at an alarming rate. The second wave of the pandemic is upon us, and it is now deadlier than the first. One could not be blamed for concluding that the situation is hopeless.

In mid-November of 2020, however, positive news emerged from pharmaceutical companies working to develop a vaccine. Several vaccines are showing promising results from Phase-3 clinical trials (up to 95\% effectiveness, National Institute of Health, 2020). Widespread vaccination campaigns may be essential to ending the pandemic, but mere news of the vaccines may begin to mitigate the virus even before they are administered. To clarify, we argue that maintaining hope during the pandemic is critical to remaining committed to public health directive aimed at mitigating the virus. Indeed, fatalism toward COVID-19 has been found to reduce intentions to practice social distancing, wear a mask, and staying away from public gathering (e.g., Jimenez et al., 2020). Thus, by reducing fatalism and promoting the belief that this virus can be overcome with coordinated action, hope for the vaccine may indirectly increase efforts to mitigate the virus with the existing (non-vaccine) public health measures. Moreover, hope for a vaccine against COVID-19 may be equally important in mitigating the mental-health burden of the pandemic (Salari et al., 2020; Xiong et al., 2020). Given that fatalism is associated with depression and general emotional distress (Hayes et al., 2016; Zimbardo \& Boyd, 1999), 
hope for a vaccine may also promote emotional resilience and well-being. The purpose of this research was to test these ideas.

\section{Overview of the Research}

We report two studies that build upon previous research investigating fatalism toward COVID-19 (Akessen et al., 2020; Hayes \& Clerk, 2020; Jimenez et al., 2020; Lifshin et al., 2020). Our primary expectation was that promoting hope for a vaccine would increase mitigation intentions and emotional well-being by reducing COVID-19 fatalism. We therefore aimed to momentarily manipulate hope for a vaccine and tested for corresponding effects. In Study 1 participants read a short article that either promoted or undermined hope for the vaccine, and in Study 2 participants read promising news in vaccine development. In both studies, we measured COVID-19 fatalism, behavioral intentions to mitigate the virus by following public health guidelines (i.e., mitigation intentions), and emotional well-being. We hypothesized that exposure to information that promotes hope for the vaccine (vs. undermines hope or vs. no information) would reduce fatalism and thereby increase mitigation intentions and emotional well-being.

In keeping with open science practices, we report all measures and manipulations, explain how sample size was determined, and report all data exclusions. Full study materials are available online at https://osf.io/uj6d2/.

Importantly, we expected the causal effects of our manipulations of vaccine-hope to emerge above and beyond any influence of demographics (e.g., income), ideology (e.g., political orientation) or personality (e.g., self-esteem). Ample evidence exists for the relationship between these extraneous variables and our variables of interest (e.g., Gollwitzer et al., 2020a; Rossi et al., 2020; Taylor et al., 2020; Weill et al., 2020). We aimed to test the effect of hope for a 
vaccine above and beyond these influences. Thus, we covaried for these extraneous factors in all our analyses (see SOM for analyses without the covariates).

\section{Study 1}

Study 1 was conducted on May $16^{\text {th }}, 2020$ (toward the end of the first wave). The purpose of the study was to examine the effect of hope for a COVID-19 vaccine on intentions to follow mitigation protocols and emotional well-being. We expected hope for the vaccine to influence these variables by affecting COVID-19 fatalism (cf., Hayes \& Clerk, 2020). To gauge the causal effect of hope, we aimed to momentarily manipulate participants' hope for the vaccine by randomly assigning them to read one of two short opinion pieces. In the high hope condition, participants read an article arguing that a vaccine will eventually be deployed and will return life to normal. The article was hopeful but not unrealistic, arguing that it will still be some time (between 12 and 18 months) before a vaccine is ready for distribution. By contrast, in the low hope condition, participants read an article that challenged the viability of a CIVID-19 vaccine and the likelihood that it would be available anytime soon. It also argued that even if a vaccine is eventually developed, it will likely not be successful in stopping the pandemic (see SOM for full text of the articles).

The experimental manipulation of hope aimed to maximize the separation between conditions by promoting vs. undermining hope for the vaccine. A comparison of these conditions will serve our purpose of examining the causal effect of hope on our dependent variables, but cannot specify which condition is most responsible for producing effects (e.g., did the high hope article increase mitigation intentions or did the low hope article reduce it?). We therefore included a third group of participants who read no article to gauge baseline scores on the 
dependent variables. We suspected scores in this condition would fall somewhere in between the high and low hope conditions.

Overall, we hypothesized that the high (vs. low) hope condition will increase behavioral intentions to follow public health advice (i.e., mitigation intentions) and emotional well-being by reducing COVID-19 fatalism.

\section{Method}

\section{Participants and Design}

To determine minimum sample size requirements to confidently test our hypotheses, we focused on our omnibus analyses and sought $80 \%$ power (with an alpha of .05) to detect a small effect (i.e., $f^{2}=.02 ; d=.20$ ). Using $\mathrm{G}^{*}$ Power (Faul et al., 2007), we estimated a sample size of 485 to detect an effect size of $f^{2}=.02$ in a linear multiple regression with two test predictors (to code for our 3 experimental conditions) and 10 total predictors (to account for 8 covariates). To ensure that we exceeded this number even after data exclusions, we collected an original sample of 1250 participants, who were recruited online through Mturk.

Participants were randomly assigned to one of three conditions in a between-subjects design. The only requirement for participation was United States residence. Exclusions included 220 participants who failed an attention check item asking them to leave a question blank (i.e., Please do not answer this question, it is here to see if you are paying attention.), 97 who did not correctly answer at least three (of five) reading comprehension questions about the contents of the article that they read, ${ }^{1}$ and 20 others who did not complete all dependent variables and covariates for our analyses. The total number of participants after exclusions was 903 (high hope $n=282$, low hope $n=287$, control $n=334)$. Participants ranged in age from 18 to 77 ( $M_{\text {age }}=38.9$, 
$\left.S D_{\text {age }}=13.6\right)$, and gender balance was roughly equal $($ female $=465$, male $=427$, other $=5$, prefer not to disclose $=6$ ).

\section{Measures and Procedure}

This study was reviewed by an institutional research ethics board and was deemed to pose no more than minimal risk. Participants were informed that the study was an investigation of personality, attitudes, and opinions. Upon consenting to participate, respondents began by completing a series of demographic questions, followed by three brief personality questionnaires.

Demographic and Personality Covariates. Demographic items included age, gender, household income, education, and political orientation (among others, see SOM for complete list of demographic items). We measured three personality factors that seemed like plausible candidates for influencing fatalism. Specifically, we assessed self-esteem (Rosenberg, 1965) and trait sensitivity to rewards and punishments (BAS and BIS; Carver \& White, 1994) given that these variables influence reactions to threat (see Jonas et al., 2014; Pyszczynski et al., 2004). These covariates were examined and found to be significant predictors of COVID-19 fatalism and/or behavioral intentions to support mitigation efforts in a previous study using similar methodology (see Hayes \& Clerk, 2020, online supplement available at https://osf.io/sx7g2/).

Vaccine Hope Manipulation. To manipulate hope for the vaccine, participants were randomly assigned to one of three conditions. In the control condition, participants read no message and simply proceeded to a series of questions related to their attitudes and opinions about the COVID-19 pandemic. By contrast, those in the high hope and low hope conditions read a brief opinion piece before proceeding to these questions. The essays began with a threatening paragraph outlining the severity of the COVID-19 pandemic: 
COVID-19 is a killer. It has already killed more than 300,000 people worldwide and will likely kill many hundreds of thousands more. Making things worse, it is an invisible killer. We cannot see it, and we cannot even know when we have it on our hands. People around the world have been trying to mitigate the spread of coronavirus for over two months (longer in China and parts of Asia), but at a global level, the spread of COVID19 shows no sign of stopping.

For participants in the high hope condition, the essay proceeded to argue that a vaccine will be able to stop the pandemic and return things to normal. The underlying message was that the pandemic is only temporary, and people will be able to control/eliminate COVID-19 just as we have done with other deadly diseases (e.g., smallpox). By contrast, participants in the low hope condition read an article that began with the same opening paragraph, but instead argued that the quick development of a vaccine is unlikely and noted that a vaccine has never been able to eradicate a coronavirus. The message was that a vaccine will not save humanity from COVID19, but rather the virus is here to stay (see SOM for the full text of conditions).

Participants in the high and low hope conditions then proceeded to complete five simple reading comprehension questions that were included to ensure adequate processing of the message. Four of these questions were multiple-choice, whereas one was an open-ended item asking participants to indicate the overall theme of the article. The open-ended question was checked for quality and marked "correct" if it appeared minimally related to the content of the article and was not simply copied text from the article. Only participants who correctly answered at least three of the five reading comprehension questions were retained for data analyses.

Specific Worries. Next, participants completed a 9-item scale assessing their specific worries related to the COVID-19 crisis. These items were included for exploratory purposes (cf., 
Hayes \& Clerk, 2020). They assessed worries about death and finances for the self, close others, and strangers, as well as worries about the loss of freedom and concern for the economy (see SOM for complete list of items).

Fatalism toward COVID-19. Participants next completed a 12-item scale assessing fatalism toward COVID-19 (originally adapted from Zimbardo \& Boyd, 1999 and revised from Hayes \& Clerk, 2020; $\alpha=.88$; see SOM for complete item-details). The scale consisted of nine positively keyed items ("Since whatever will be will be, it doesn't really matter what we do to try to stop COVID-19.") and three negatively-keyed items (e.g., "What we do now to fight covid-19 will mater in the long run."). Participants rated their agreement with each item using a 7-point Likert scale ( 1 = strongly disagree; 7 = strongly agree $)$.

Mitigation Intentions. Immediately after the fatalism questionnaire, we included a 12item scale assessing behavioral intentions to mitigate the virus by following public health guidelines (revised from Hayes \& Clerk, 2020; $\alpha=.90$, see SOM for complete item-details). The scale consisted of 10 positively keyed items (e.g., "I plan to stay isolated for as long as it is required;" "I plan to wear a mask when in public.") and two negatively-keyed items (e.g., "I am willing to bend the rules of social distancing."). Participants were asked to indicate how they intended to behave "within the coming weeks" by rating their agreement with each item on a 7point scale.

Emotional Well-Being. Finally, participants completed a brief emotion measure to assess emotional well-being. The measure consisted of 20 items assessing four different emotions (5-items each: anxiety, fear, depression, and felt-security; $\alpha=.95$; see SOM for complete item-details). Participants rated the extent to which they were currently experiencing 
these emotions using a 7 -point scale $(1=$ not at all; $7=$ very much). Items representing anxiety, fear, and depression were reverse-scored in the computation of emotional well-being.

Upon completion, participants completed a self-affirmation mood-repair exercise before being fully debriefed and thanked for their participation.

\section{Results}

Deidentified data and analysis script for all analyses reported below are available online at https://osf.io/uj6d2/.

\section{Test of Total Effects}

To test our hypothesis about the effect of experimental condition on COVID-19 fatalism, mitigation intentions, and emotional well-being, we conducted hierarchical multiple regression analyses on each of these dependent variables. We included demographic and personality factors in Step 1, and the vaccine hope-manipulation (dummy coded) ${ }^{2}$ in Step 2 (see Table 1 for full results). In summary, these results show a significant effect of hope condition over and above the demographic and personality factors on COVID-19 fatalism, $\Delta R^{2}=.03, F(2,892)=17.62$, $p<$ .001 , and emotional well-being, $\Delta R^{2}=.01, F(2,892)=5.23, p=.005$, but not for mitigation intentions, $\Delta R^{2}=.00, F(2,892)=1.06, p=.348$. Table 2 displays the means and standard deviations for all three dependent variables across conditions.

Pairwise comparisons on the fatalism scores show that the high hope condition significantly reduced fatalism relative to the low hope condition, $t(892)=-5.92, p<.001, d=-$ .50 , and relative to the control condition, $t(892)=-2.72, p=.005, d=-.22$. Regarding emotional well-being, the high hope condition significantly increased well-being relative to the low hope condition, $t(892)=3.22, p=.001, d=.27$, but not relative to the control condition, $t(892)=1.42$, $p=.155, d=.12$. 


\section{Test of Indirect Effects}

Although the vaccine thought-manipulation did not significantly affect virus mitigation intentions in the total effects analysis, our main hypothesis was that influencing hope for the vaccine would have an indirect effect on mitigation intentions and emotional well-being by virtue of influencing COVID-19 fatalism (cf., Hayes \& Clerk, 2020). Given the strong effect of the manipulation on fatalism, we proceeded to test our expectations regarding the indirect effects using Hayes' (2018) PROCESS macro.

Accordingly, we first regressed mitigation intentions on vaccine hope condition (dummycoded as above) through the mediator of COVID-19 fatalism (Model 4, 5000 bootstrap resamples, include all covariates from above analyses). As expected, this analysis showed a significant indirect effect of hope for the vaccine on mitigation intentions. This effect emerged regardless of whether the boost condition is compared to the threat condition, $b=.16,95 \% \mathrm{CI}$ $[.098, .233]$ or to the control condition, $b=.07,95 \%$ CI $[.018, .130]$. Next, we conducted the same PROCESS analysis on emotional well-being. As hypothesized, we found a significant indirect effect of hope for the vaccine on well-being regardless of whether we compared to boost condition to the threat condition, $b=.14,95 \% \mathrm{CI}[.086, .202]$ or to the control condition, $b=.06$, 95\% CI $[.015, .114]$ (see Figure 1 for a full path model).

\section{Discussion}

The results of Study 1 support our hypotheses regarding the effect of hope for a COVID19 vaccine on mitigation intentions and emotional well-being. As expected, these effects emerged by virtue of influencing COVID-19 fatalism, which negatively predicted both mitigation intentions and emotional well-being. Thus, high hope for the vaccine indirectly 
increased participants' intentions to following public health mitigation protocols and increased their overall emotional well-being.

Importantly, the effects of experimentally heightened hope for the vaccine emerged regardless of the comparison condition. Although the effects were strongest when comparing the high hope to the low hope condition, the effects were nevertheless detectable in comparison to the baseline control condition. Moreover, the effects were independent of demographic, ideological, and personality variables measured in the study.

\section{Study 2}

Study 2 sought to replicate and extend the results of Study 1. It was conducted on November 20-21, 2020, within days of news that vaccines by Pfizer and Moderna were highly effective in preventing COVID-19 and nearly ready to be approved for widespread distribution. Naturally, this news increased hope that a vaccine can stop the ongoing pandemic. We therefore sought to examine the influence of this positive vaccine news on mitigation intentions and emotional well-being.

Accordingly, the design of Study 2 was nearly identical to that of Study 1, except all participants read a short article emphasizing the severity of the pandemic by highlighting current daily death-totals around the world and specifically in the United States. Participants in the vaccine news condition, however, were given additional information about the forthcoming vaccines, whereas those in the control condition were not. We expected the vaccine news (vs. control) to reduce fatalism and consequently increase mitigation intentions and emotional wellbeing.

As an added feature to Study 2, we also asked participants about their intentions to take the vaccine when it becomes available. This item was included for exploratory purposes. 
However, consistent with our expectations regarding mitigation intentions more generally, we tentatively expected the vaccine news manipulation to increase intentions to take the vaccine by virtue of reducing COVID-19 fatalism.

\section{Method}

\section{Participants and Design}

We conducted an a priori power analysis using the effect size from Study 1 to determine minimum sample size requirements for Study 2. We focused on the primary effect of vaccine hope on COVID-19 fatalism to conduct our analysis. Study 1 showed small-to-medium effects of hope for the vaccine (high vs. low hope, $d=-.50$; high hope vs. control, $d=-.22$ ). We used the average of these effects to determine sample size in Study $2\left(d=.36\right.$ or $\left.f^{2}=03\right)$ and sought at least $80 \%$ power (with an alpha of .05 ) to detect this effect in a multiple regression analysis with 9 predictors (the focal predictor with 8 covariates). This analysis yielded an estimate of $N=264$. As in Study 1, we oversampled to ensure a final sample size of at least 264 after data exclusions. Participants were 440 people recruited online through Mturk. They were randomly assigned to one of two conditions in a between-subjects design. The requirements for participation were United States residence, 90\% HIT acceptance rate, a researcher-created qualification obtained from participation in a large-scale sample collected 2-3 weeks earlier $(N$ $\sim=2,000) .{ }^{3}$ Exclusions included 12 participants who failed an attention check item asking them to leave a question blank (i.e., Please do not answer this question, it is here to see if you are paying attention.), 22 who did not offer a quality response to an item asking them to summarize the content of the opinion article (same criterion as Study 1), and 12 others who failed to complete all dependent variables and covariates for our analyses. The total number of participants after exclusions was 394 (vaccine new $n=181$, control $n=213$ ). Participants ranged in 
age from 19 to $83(M=43.1, S D=13.3)$, and gender balance was roughly equal (female $=216$, male $=171$, other $=4$, prefer not to disclose $=3$ ).

\section{Measures and Procedure}

Materials and procedures were nearly identical to Study 1, except for the nature of the vaccine hope manipulation.

Vaccine Hope Manipulation. To manipulate hope for the vaccine, participants were randomly assigned to read one of two opinion essays. All participants read the same opening paragraph that emphasized the severity of the pandemic by listing the current death-totals and rate of death, both worldwide and specifically in the United States. Participants in the vaccine news condition read an additional paragraph that offered real news of the forthcoming vaccines (see SOM for complete text of the articles).

Dependent Variables. The dependent variables of fatalism and emotional well-being were the same as Study 1, but we made a few minor items changes to the mitigation intentions scale to keep up with current mitigation practices (e.g., "stay isolated" was revised to "minimize social contacts;" see SOM for complete item details). Most significantly, toward the end of the study, we included a single item asking participants to indicate how likely they are to take a COVID-19 vaccine. The item read "When a vaccine is finally available to the masses, assuming it is readily available to you (at little/no cost), what is your attitude toward taking it?" Participants responded using a 5-point scale (where $1=I$ will certainly not take the vaccine, $2=I$ am unlikely to take the vaccine, $3=$ I am unsure about taking the vaccine, $4=$ I am likely to take the vaccine, $5=$ I will certainly take the vaccine $)$.

Finally, participants completed a self-affirmation mood-repair exercise, were presented with a debriefing letter, and were thanked for their participation. 


\section{Results}

Consistent with Study 1, deidentified data and analysis script for all analyses reported below are available at https://osf.io/uj6d2/.

\section{Test of Total Effects}

We began as we did with Study 1, by testing for total effects of vaccine news on COVID19 fatalism, mitigation intentions, emotional well-being, and behavioral intentions to get vaccinated. Accordingly, we conducted hierarchical multiple regression analyses on each of these dependent variables, with demographic and personality factors included in Step 1, and the vaccine news manipulation (vaccine news $=1$, no news $=0$ ) in Step 2 (see Table 3 for full results). In summary, these results show a significant effect of vaccine new over and above the demographic and personality factors on COVID-19 fatalism, $\Delta R^{2}=.01, F(1,384)=4.76, p=$ .030 , and vaccination intentions, $\Delta R^{2}=.01, F(1,384)=5.31, p=.022$, such that reading about the vaccine news reduced fatalism $(d=-.22)$ and increased intentions to get vaccinated $(d=.23)$. The total effect of vaccination news was not significant with respect to general mitigation intentions, $\Delta R^{2}=.01, F(1,384)=2.72, p=.100$, or emotional well-being, $\Delta R^{2}=.00, F(1,384)=$ $0.43, p=.513$. Table 4 displays the means and standard deviations for all four dependent variables across conditions.

\section{Test of Indirect Effects}

To test our hypotheses regarding the indirect effects of vaccine news through COVID-19 fatalism, we next conducted a series of PROCESS analyses (Hayes, 2018) on each of the other dependent variables (Model 4, 5000 bootstrap resamples). Consistent with hypotheses, these analyses showed significant indirect effects of vaccine news on general mitigation intentions, $b=$ $.09,95 \%$ CI $[.008, .179]$, and vaccination intentions, $b=.07,95 \%$ CI $[.005, .140]$, but the 
indirect effect on emotional well-being was not quite significant, $b=.03,95 \%$ CI [ -.001, .067] (see Figure 1 for a full path model).

\section{Discussion}

The results of Study 2 are consistent with observations from Study 1 and supportive of our hypotheses. Vaccine news reduced COVID-19 fatalism, which in turn increased general mitigation intentions and specific intentions to get vaccinated against COVID-19. Although the vaccine news condition did not significantly affect emotional well-being, COVID-19 fatalism was negatively associated with emotional well-being and the indirect effect of vaccine news on emotional well-being through fatalism was in the predicted direction. Thus, although the results do not support our hypothesis regarding emotional well-being with $95 \%$ certainty, they are nevertheless consistent with expectations.

\section{General Discussion}

This research shows a causal effect of hope for a COVID-19 vaccine on mitigation intentions and mental health. The evidence suggests that increasing hope for a vaccine promotes adherence to public health advice (e.g., mask wearing) and emotional well-being by reducing fatalism toward the virus. These results have important implications for improving both physical and mental health during the pandemic.

Regarding physical health, efforts to save lives by persuading people to follow mitigation protocols have often been met with fierce resistance among some demographic groups (e.g., political conservatism; Gollwitzer et al., 2020a). The current studies show an effect of hope for the vaccine on mitigation intentions regardless of demographic factors. Thus, promoting hope for a vaccine may be an indirect way to increase adherence to mitigation protocols. Indeed, Study 2 suggests that hope for a vaccine may even increase people's willingness to ultimately get 
vaccinated. Given fear that too many people will forego vaccination (Cornwall, 2020), any effect that might nudge people toward vaccination will help to reduce the prevalence of the virus in the population and thereby save many lives. Available evidence shows a strong correlation between behavioral intentions and actual mitigation behavior during this pandemic (Gollwitzer et al., 2020b; Hagger et al., 2020), so these results are highly encouraging.

Regarding mental health, maintaining hope that this pandemic will eventually end is of paramount importance to the well-being of nearly everyone on the planet. Although a vaccine is not the only way to end to this pandemic, it remains the most hopeful option. The current research suggest that guarding this is an important factor in maintaining emotional resilience while the pandemic continues.

Although the current research offers promising implications, a note of caution may be justified. Officials at the World Health Organization have sought to temper expectations for a vaccine, in part to convince people that continued adherence to mitigation protocols will remain necessary for some time to come (Taylor, 2020). The current research may suggest that this strategy will backfire by undermining hope. Our results show that reducing hope can consequently reduce mitigation intentions by promoting fatalism. However, it also remains possible that falsely inflating hope could backfire for the same reason. Placing too much hope on a vaccine to end the pandemic may lead to extreme disappointment if vaccines are not ultimately successful. This might trigger an even greater sense of fatalism and may consequently lead people to altogether abandon mitigation efforts and experience a mental health crisis. Careful consideration should thus be given to promoting hope without falsely inflating it.

\section{Conclusion}


Maintain hope in the face of extreme hardship is an important factor in behavioral and emotional resilience. The current research shows that hope for a vaccine in the fight against COVID-19 can reduce fatalism, increase emotional well-being, and increase people willingness to follow public health advice and get vaccinated. Importantly, these effects are independent of any actual effect that the vaccine will have on preventing the spread of the virus. Merely maintaining hope that the virus can be stopped is critically important in preventing people from fatalistically abandoning efforts to fight the pandemic. In this sense, the prospect of a forthcoming vaccine may be nearly as important as the vaccine itself. 


\section{Footnotes}

${ }^{1}$ This exclusion criterion could only apply to the high and low hope groups because participants in the control condition did not read an article.

${ }^{2}$ Dummy1 compared high hope to low hope; dummy2 compared high hope to control.

${ }^{3}$ This later inclusion criterion was added to minimize data-exclusions (and thus resource waste) because these participants had already been screened for random responding. Inclusion criteria for this larger study were United States residence and 90\% HIT acceptance rate. 


\section{Author Contributions}

J. Hayes and L. Clerk developed the study concept. J. Hayes designed the study, and L. Clerk provided critical feedback. Data collection, analysis, and interpretation was performed by J.

Hayes. L. Clerk performed an extensive review of the literature. J. Hayes drafted the manuscript, and L. Clerk provided critical revisions. Both authors approved the final version of the manuscript for submission. 


\section{References}

Akesson, J., Ashworth-Hayes, S., Hahn, R. W., Metcalfe, R., \& Rasooly, I. (2020). Fatalism, beliefs, and behaviors during the COVID-19 pandemic [Preprint]. National Bureau of Economic Research. Working Paper No. w27245, http://doi.org/10.3386/w27245

Carver, C. S., \& White, T. L. (1994). Behavioural inhibition, behavioural activation, and affective responses to impending reward and punishment: The BIS/BAS scales. Journal of Personality and Social Psychology, 67(2), 319-333. http://www.doi.org/10.13072/midss.536

Cornwall, W. (2020, June 30). Just 50\% of Americans plan to get a COVID-19 vaccine. Here's how to win over the rest. Science Magazine.

https://www.sciencemag.org/news/2020/06/just-50-americans-plan-get-covid-19-vaccinehere-s-how-win-over-rest

Faul, F., Erdfelder, E., Lang, A., \& Buchner, A. (2007). G*Power 3: A flexible statistical power analysis program for the social, behavioral, and biomedical sciences. Behavior Research Methods, 39(2), 175-191. https://doi.org/10.3758/BF03193146

Gollwitzer, A., Martel, C., Brady, W. J., Parnamets, P., Freedman, I. G., Knowles, E. D., \& Van Bavel, J. J. (2020a). Partisan differences in physical distancing are linked to health outcomes during the COVID-19 pandemic. Nature Human Behaviour, 4, 1186-1197. doi:10.1038/s41562-020-00977-7

Gollwitzer, A., Martel, C., Marshall, J., Hohs, J. M., \& Bargh, J. A. (2020b). Connecting selfreported social distancing to real-world behavior at the individual and U.S. State Level. [Preprint]. PsyArXiv. doi:10.31234/osf.io/kvnwp 
Hagger, M. S., Smith, S. R., Keech, J. J., Moyers, S. A., \& Hamilton, K. (2020). Predicting social distancing intention and behavior during the COVID-19 pandemic: An integrated social cognition model. Annals of behavioral medicine : a publication of the Society of Behavioral Medicine, 54(10), 713-727. https://doi.org/10.1093/abm/kaaa073

Hayes, A. F. (2018). Introduction to mediation, moderation, and conditional process analysis: A regression-based approach (2 ${ }^{\text {nd }}$ ed.). New York: Guilford Press.

Hayes, J., \& Clerk, L. (2020). Fatalism in the fight against COVID-19: Implications for mitigation and mental health [Preprint]. http://doi.org/10.31219/osf.io/t6zmv

Hayes, J., Ward, C. L. P., \& McGregor, I. (2016). Why bother? Death, failure, and fatalistic withdrawal from life. Journal of Personality and Social Psychology, 110(1), 96-115. http://doi.org/10.1037/pspp0000039

Jimenez, T., Restar, A., Helm, P. J., Cross, R. I., Barath, D., \& Arndt, J. (2020). Fatalism in the context of COVID-19: Perceiving coronavirus as a death sentence predicts reluctance to perform recommended preventive behaviors. SSM - Population Health, 11, 100615. https://doi.org/10.1016/j.ssmph.2020.100615

Jonas, E., McGregor, I., Klackl, J., Agroskin, D., Fritsche, I., Holbrook, C., Nash, K., Proulx, T., \& Quirin, M. (2014). Advances in experimental social psychology. In J. M.Olson \& M. P.Zanna (Eds.), Threat and defense: From anxiety to approach (pp. 219-286). Academic Press.

Lifshin, U., Mikulincer, M., \& Kretchner, M. (2020). Motivated helplessness in the context of the COVID-19 pandemic: Evidence for a curvilinear relationship between perceived ability to avoid the virus and anxiety. Journal of Social and Clinical Psychology, 39, 479497. https://doi.org/10.1521/jscp.2020.39.6.479 
National Institute of Health. (2020, November 16). Promising Interim Results from Clinical Trial of NIH-Moderna COVID-19 Vaccine [News release]. https://www.nih.gov/newsevents/news-releases/promising-interim-results-clinical-trial-nih-moderna-covid-19$\underline{\text { vaccine }}$

Pyszczynski, T., Greenberg, J., Solomon, S., Arndt, J., \& Schimel, J. (2004). Why do people need self-esteem? A theoretical and empirical review. Psychological Bulletin, 130(3), 435-468. http://www.doi.org/10.1037/0033-2909.130.3.435

Rosenberg, M. (1965). Society and the adolescent self-image. Princeton University Press.

Rossi, A., Panzeri, A., Pietrabissa, G., Manzoni, G. M., Castelnuovo, G., \& Mannarini, S. (2020). The anxiety-buffer hypothesis in the time of COVID-18: When self-esteem protects from the impact of loneliness and fear on anxiety and depression. Frontiers in Psychology, 11, 2177. doi:10.3389/fpsyg.2020.02177

Salari, N., Hosseinian-Far, A., Jalali, R., Vaisi-Raygani, A., Rasoulpoor, S. Mohammadi, M., Rasoulpoor, S., ; Khaledi-Paveh, B. (2020). Prevalence of stress, anxiety, depression among the general population during the COVID-19 pandemic: A systematic review and meta-analysis. Globalization and Health, 16, (57). http://doi.org/10.1186/s12992-020$\underline{00589-\mathrm{w}}$

Taylor, A. (2020). WHO tempers prospect of a coronavirus vaccine with warning of a long fight to come. The Washington Post. https://www.washingtonpost.com/world/2020/11/16/whovaccine-moderna-pfizer/

Taylor, S., Landry, C., Paluszek, M. M., \& Asmundson, G. J. G. (2020). Reactions to COVID19: Differential predictors of distress, avoidance, and disregard for social distancing. Journal of Affective Disorders, 277, 94-98. doi:10.1016/j.jad.2020.08.002 
Weill, J. A., Stigler, M., Deschenes, O., \& Spingborn, M. R. (2020). Social distancing responses to COVID-19 emergency declarations strongly differentiated by income. Proceedings of the National Academy of Science, 117, 33. doi:10.1073/pnas.2009412117

Worldometer. (2020). Covid-19 coronavirus pandemic [Infograph]. Worldometer.com. http://www.worldometers.info/coronavirus/

Xiong, J., Lipsitz, O., Nasri, F., Lui, L. M. W., Gill, H., Phan, L., Chen-Li, D., Iacobucci, M., Ho, R., Majeed, A., \& McIntyre, R. S. (2020). Impact of COVID-19 pandemic on mental health in the general population: A systematic review. Journal of Affective Disorders, 277, 55-64. doi:10.1016/j.jad.2020.08.001

Zimbardo, P. G., \& Boyd, J. N. (1999). Putting time in perspective: A valid, reliable individualdifferences metric. Journal of Personality and Social Psychology, 77(6), 1271-1288. http://www.doi.org/10.1037/0022-3514.77.6.1271 


\section{Table 1}

Multiple Regression Results Predicting Fatalism Toward COVID-19, Behavioral Intentions to Support Mitigation Efforts, and Emotional Well-Being from Demographics, Personality Factors, and Experimental Condition in Study 1.

\begin{tabular}{|c|c|c|c|c|c|c|}
\hline \multirow[b]{2}{*}{ Predictor } & \multicolumn{2}{|c|}{$\begin{array}{c}\text { COVID-19 } \\
\text { Fatalism }\end{array}$} & \multicolumn{2}{|c|}{$\begin{array}{l}\text { Mitigation } \\
\text { Intentions }\end{array}$} & \multicolumn{2}{|c|}{$\begin{array}{c}\text { Emotional } \\
\text { Well-Being }\end{array}$} \\
\hline & $\beta$ & $t$ & $\beta$ & $t$ & $\beta$ & $t$ \\
\hline Age & -.04 & -1.29 & .09 & $2.91^{* *}$ & -.01 & -0.48 \\
\hline Male gender & -.01 & -0.36 & -.06 & $-1.96^{\dagger}$ & .04 & 1.54 \\
\hline Income & -.09 & $-3.07^{* *}$ & .06 & $1.95^{\dagger}$ & .11 & $3.99^{* * *}$ \\
\hline Education & .07 & $2.36^{*}$ & .11 & $3.58^{* * * *}$ & -.07 & $-2.80^{* *}$ \\
\hline Political Conservatism & .25 & $8.45^{* * *}$ & -.28 & $-9.29^{* * *}$ & -.04 & -1.72 \\
\hline Self-Esteem & -.42 & $-12.54^{* * *}$ & .23 & $6.78^{* * *}$ & .55 & $18.81^{* * *}$ \\
\hline BAS Sensitivity & .13 & $4.28^{* * * *}$ & .10 & $3.15^{* *}$ & -.09 & $-3.30^{* *}$ \\
\hline BIS Sensitivity & -.20 & $-5.84^{* * *}$ & .26 & $7.41^{* * *}$ & -.16 & $-5.28^{* * *}$ \\
\hline C1 (Low vs. High Hope) & -.20 & $-5.92^{* * *}$ & .05 & 1.41 & .10 & $3.22^{* *}$ \\
\hline C2 (Control vs. High Hope) & -.09 & $-2.72^{* *}$ & .02 & 0.43 & .04 & 1.42 \\
\hline
\end{tabular}

Note. Gender coded male $=1$, non-male (including female, other, and prefer not to disclose) $=0$; $\mathrm{C} 1$ represents effect of high (vs. low) hope condition; C2 represents effect of high hope (vs. control condition.

${ }^{\dagger} p<.100 *{ }^{*} p<.050 *{ }^{* *} p<.010 * * * * 001$ 


\section{Table 2}

Means and standard deviations for dependent variables by experimental conditions in Study 1.

\begin{tabular}{lccc} 
& \multicolumn{3}{c}{ Experimental Condition } \\
\cline { 2 - 4 } Dependent Variable & High Hope & Low Hope & Control \\
\hline Fatalism & $2.90_{\mathrm{a}}(1.21)$ & $3.40_{\mathrm{b}}(1.05)$ & $3.12_{\mathrm{c}}(1.20)$ \\
Mitigation Intentions & $5.40_{\mathrm{a}}(1.06)$ & $5.28_{\mathrm{a}}(1.17)$ & $5.36_{\mathrm{a}}(1.10)$ \\
Emotional Well-Being & $5.03_{\mathrm{a}}(1.25)$ & $4.76_{\mathrm{b}}(1.37)$ & $4.91_{\mathrm{a}, \mathrm{b}}(1.33)$ \\
\hline
\end{tabular}

Note: Means represent values controlling for all covariates in the model. Standard deviations are displayed in parentheses. Means that do not share a subscript are different at the $p<.05$ level. 


\section{Table 3}

Multiple Regression Results Predicting Fatalism Toward COVID-19, Behavioral Intentions to Support Mitigation Efforts, Emotional Well-Being, and Intentions to Take a COVID-19 Vaccine from Demographics, Personality Factors, and Experimental Condition in Study 2.

\begin{tabular}{|c|c|c|c|c|c|c|c|c|}
\hline \multirow[b]{2}{*}{ Predictor } & \multicolumn{2}{|c|}{$\begin{array}{c}\text { COVID-19 } \\
\text { Fatalism }\end{array}$} & \multicolumn{2}{|c|}{$\begin{array}{l}\text { Mitigation } \\
\text { Intentions }\end{array}$} & \multicolumn{2}{|c|}{$\begin{array}{c}\text { Emotional } \\
\text { Well-Being }\end{array}$} & \multicolumn{2}{|c|}{$\begin{array}{l}\text { Vaccination } \\
\text { Intentions }\end{array}$} \\
\hline & $\beta$ & $t$ & $\beta$ & $t$ & $\beta$ & $t$ & $\beta$ & $t$ \\
\hline Age & -.13 & $-2.79^{* *}$ & .16 & $3.62^{* * * *}$ & -.06 & -1.53 & .06 & 1.14 \\
\hline Male gender & -.08 & $-1.81^{\dagger}$ & -.05 & -1.14 & -.00 & -0.01 & .05 & 1.06 \\
\hline Income & -.01 & -0.14 & .03 & 0.71 & .03 & 0.85 & .06 & 1.11 \\
\hline Education & .01 & 0.20 & -.00 & -0.05 & -.02 & -0.43 & .19 & $3.87^{* * *}$ \\
\hline Conservatism & .31 & $7.03^{* * *}$ & -.40 & $-9.33^{* * *}$ & .06 & $1.68^{\dagger}$ & -.22 & $-4.70^{* * *}$ \\
\hline Self-Esteem & -.43 & $-8.61^{* * *}$ & .25 & $5.11^{* * *}$ & .54 & $12.64^{* * *}$ & .14 & $2.54^{*}$ \\
\hline BAS & .04 & 0.77 & .17 & $3.79^{* * * *}$ & -.07 & $-1.70^{\dagger}$ & .08 & 1.62 \\
\hline BIS Sensitivity & -.17 & $-3.43^{* *}$ & .23 & $4.48^{* * * *}$ & -.27 & $-6.20^{* * *}$ & .08 & 1.40 \\
\hline Vaccine News & -.10 & $-2.18^{*}$ & .07 & 1.65 & .02 & 0.66 & .11 & $2.30^{*}$ \\
\hline
\end{tabular}

Note. Gender coded male $=1$, non-male (including female, other, and prefer not to disclose) $=0$ ${ }^{\dagger} p<.100{ }^{*} p<.050{ }^{* *} p<.010{ }^{* * *} p<.001$ 


\section{Table 4}

Means and standard deviations for dependent variables by experimental condition in Study 2.

\begin{tabular}{lcc} 
& \multicolumn{2}{c}{ Experimental Condition } \\
\cline { 2 - 3 } Dependent Variable & Vaccine News & Control \\
\hline COVID-19 Fatalism & $2.54_{\mathrm{a}}(1.05)$ & $2.75_{\mathrm{b}}(1.17)$ \\
Mitigation Intentions & $5.64_{\mathrm{a}}(1.17)$ & $5.47_{\mathrm{a}}(1.26)$ \\
Emotional Well-Being & $5.41_{\mathrm{a}}(1.23)$ & $5.36_{\mathrm{a}}(1.34)$ \\
Vaccination Intentions & $3.88_{\mathrm{a}}(1.30)$ & $3.58_{\mathrm{b}}(1.36)$ \\
\hline
\end{tabular}

Note: Means represent values controlling for all covariates in the model. Standard deviations are displayed in parentheses. Means that do not share a subscript are different at the $p<.05$ level. 


\section{Figure 1}

Path model of indirect effects of vaccine hope conditions on mitigation intentions and emotional well-being through COVID-19 fatalism in Study 1.

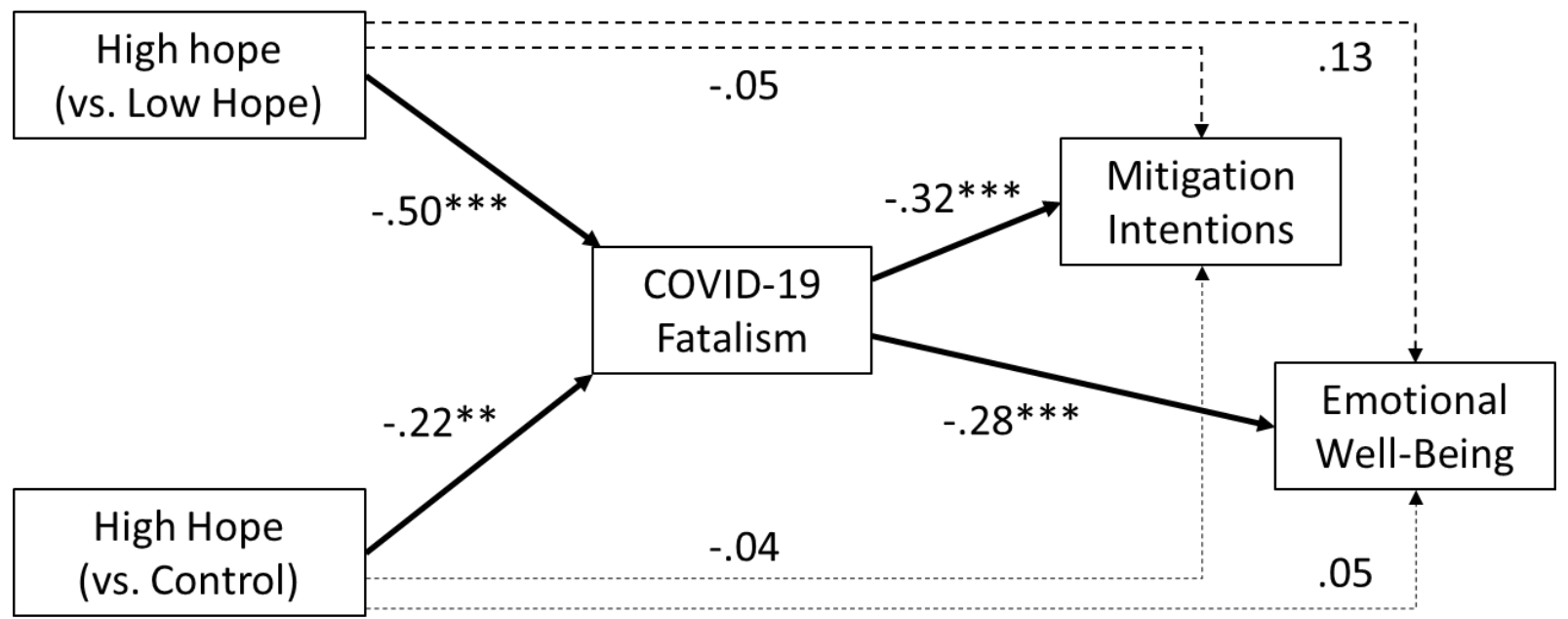

Note: Coefficients represent unstandardized regression weights.

${ }^{* *} p<.01{ }^{* * *} p<.001$ 


\section{Figure 2}

Path model of indirect effects of vaccine news on mitigation intentions, emotional well-being, and vaccination intentions through COVID-19 fatalism in Study 2.

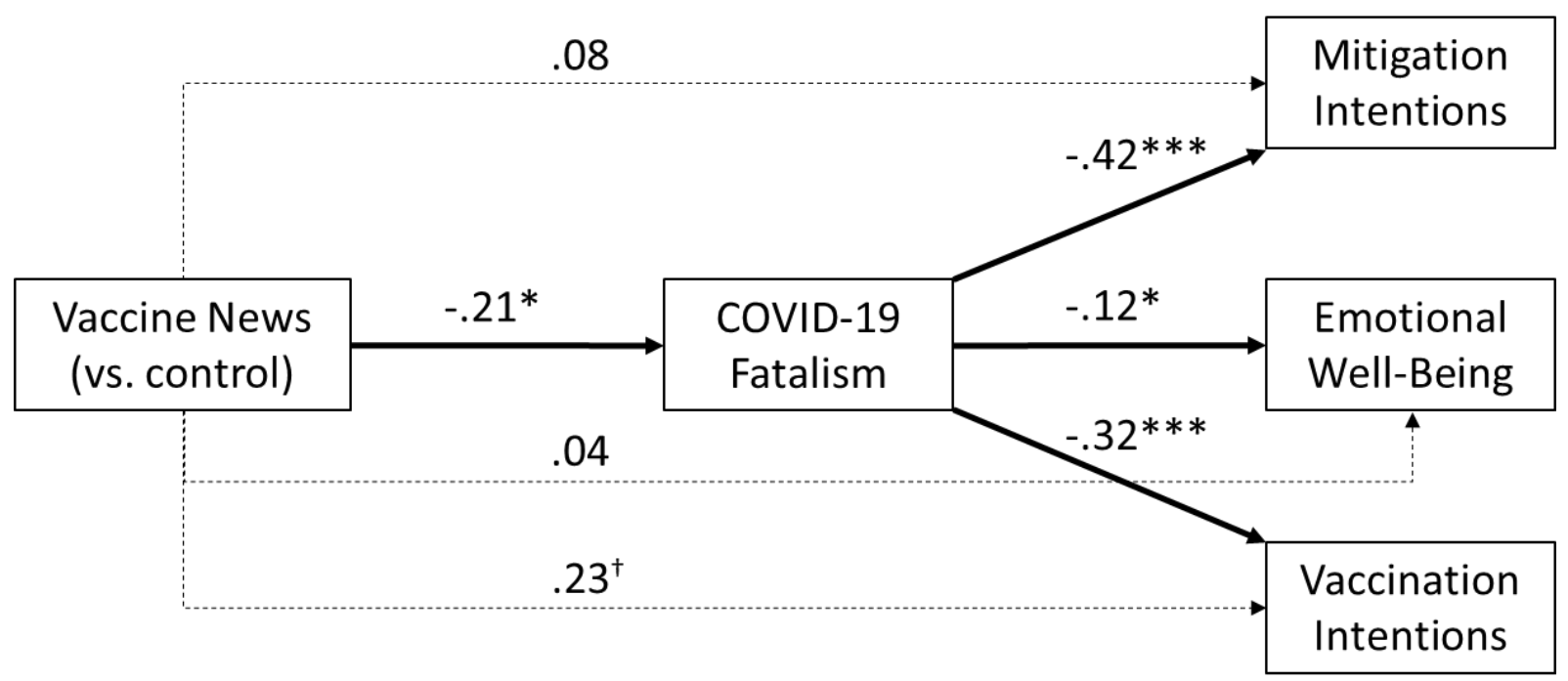

Note: Coefficients represent unstandardized regression weights.

${ }^{\dagger} p<.100{ }^{*} p<.050{ }^{* * *} p<.001$ 